\title{
PTU-114 PROGNOSTIC VALUE OF GRADING IN NEUROENDOCRINE TUMOURS
}

doi:10.1136/gut.2011.239301.242

M S Khan, 1,* F El-Khouly, ${ }^{1}$ C Toumpanakis, ${ }^{1}$ M Caplin,1 T V Luong ${ }^{1}$ Neuroendocrine Tumour Unit, London, UK; ${ }^{2}$ Department of Pathology, Royal Free Hospital, London, UK

Introduction Gastroenteropancreatic (GEP) neuroendocrine tumours (NETs) are uncommon tumours but with an increasing prevalence (35 per 100000$)$. They are generally slow-growing but have variable proliferation indices. The recent classification system of NETs proposed by the European Neuroendocrine Tumour Society (ENETS) uses Ki-67 index and/or mitotic count to assign grade (low, intermediate, high) ${ }^{1}$. We have previously demonstrated a discrepancy in grade assignment depending on whether Ki-67 or mitotic count is used ${ }^{2}$. It has been adopted into routine practice but there is limited data on the relationship between these indices and whether they have similar prognostic value. We explored the prognostic value of grading according to $\mathrm{Ki}-67$ and mitotic count in GEP NETs.

Methods 285 NET patients, 144 midgut and 141 pancreatic, had their cases reviewed. Ki-67 proliferation index, identified by staining tumour sections with MIB-1 antibody, was determined by assessing the percentage of positively staining tumour cell nuclei in 2000 tumour cells. Mitotic counts (per 10 high power fields) were evaluated in at least 40 fields in areas of highest mitotic activity. Grades (1 to 3 ) according to the new TNM classification were assigned using both indices. Slides were assessed by an experienced pathologist with expertise in NETs. Progression-free survival (PFS) and overall survival (OS) from date of diagnosis were estimated using KaplanMeier methodology and Cox Regression used for multivariate analysis.

Results 131/144 midgut and 136/141 pancreatic NETs had complete data. As previously reported, there was discordance in grade assignment (38\% and $51 \%$ respectively). Median follow-up was 42 months in midgut and 46 months in pancreatic NETs. 5-year PFS and OS for pancreatic NETs were $29 \%$ and $53 \%$ respectively. 5 -year PFS and OS for midgut NETs were $37 \%$ and $61 \%$. On univariate analysis, grade according to either $\mathrm{Ki}-67$ or mitotic count was prognostic of PFS and OS in both types of NETs. On multivariate analysis, grade according to Ki-67 was an independent prognostic indicator of PFS and OS $(\mathrm{p}<0.001)$. Grade according to mitotic count was not $(\mathrm{p}>0.16)$.

Conclusion Grade according to Ki-67 is of prognostic significance but grade assigned by mitotic count may not provide similar prognostic value based on the current classification thresholds. Further studies evaluating grading thresholds of mitotic counts and investigation of other mitotic markers are required.

Competing interests None.

Keywords carcinoid, neuroendocrine tumour, prognostic biomarkers.

\section{REFERENCES}

1. Rindi et al. TNM Staging of Midgut and Hindgut NETs: a consensus proposal including a grading system. Virchows Arch 2007;451:757-62.

2. Khan MS et al. Classification by grade in gastroenteropancreatic neuroendocrine tumours. Gut 2010;59:A111. 